\title{
Ejercicio físico como terapia no farmacológica en la artrosis de rodilla
}

\author{
Pedro José Benito Peinado*, Rocío Cupeiro Coto y Francisco Javier Calderón Montero \\ Facuitad de Ciencias de la Actividad Fisica y del Deporte. INEF, Universidad Palitéculica de Madizd, Madizi, Espaía
}

Palabras dave:

Artrosis

Enfermedad articular

Ejercicio

Actividad fisica

Fuerza

\section{R ES U M E N}

La artrosis de rodilla es una de las enfermedades articulares más frecuentes, sus sintomas principales son dolor e incapacidad fisica. La regeneración del cartílago es un tratamiento todavía en desarrollo, por lo que los tratamientos actuales se centran en aliviar los síntomas. El ejercicio físico se presenta como una alternativa u opción de tratamiento barata y efectiva. Sin embargo, todavía no está claro qué tipo de ejercicio, cantidad, intensidad, etc. son más recomendables. Por lo tanto, el objetivo de esta revisión es integrar toda la información posible de cara al diseño de programas de entrenamiento para el tratamiento de la artrosis de rodilla. Todos los artículos seleccionados tras la revisión, salvo el de Talbot et al ${ }^{1}$, mostraron mejoras significativas en el dolor de la rodilla, en la capacidad fisica o en ambas variables. Sin embargo, muchos autores obvian elementos cruciales del programa, por lo que no es posible la aplicación con fines terapéuticos o para contrastar los resultados en otras muestras.

\section{Physical exercise as non pharmacologic therapy in knee osteoarthritis}

\author{
A B S T R A C T
}

Knee osteoarthritis is one of the most frequent joint disorders, and its major symptoms are pain and physical disability. Cartilage regeneration therapies are still under development, and current treatments target pain and disability. Physical activity could be a cheap and effective therapeutic option. However, it is not yet known which types of exercise are the most beneficial, as well as its load or intensity. Therefore, the objective of this work is to integrate all the information about the design of training programs for lanee osteoarthritis treatment. All of the selected articles by Talbot and colleagues (except one), showed significant improvement in knee pain, physical performance, or both. However, many authors do not describe the main elements of the programs, so its application as a therapy or for contrasting the results is not possible.

\section{Introducción}

Dentro de los diferentes tipos de tratamientos que pueden emplearse en la intervención sobre la artrosis de rodilla, el ejercicio fisico parece el que menos efectos secundarios o secuelas produce. Sin embargo, hay bastante controversia ${ }^{2}$ sobre quê tipo de ejercicio es más adecuado y qué dosis son las más recomendables. Este trabajo pretende ayudar a definir, desde el punto de vista práctico, las características de los tratamientos utilizados en los estudios clínicos.

\section{Marco teórico}

Se calcula que la artrosis sintomática de rodilla (definida como dolor en la mayoría de los dias, además de evidencias de la

\footnotetext{
" Autor para correspondencia.

Correo electrónico: pedroj.benito@upm.es (P.J. Benito Peinado).
}

enfermedad en una radiografía de la rodilla afectada) tiene una prevalencia del $11 \%$ en los individuos mayores de 65 años ${ }^{3}$.

En España, según la Encuesta Nacional de Salud de 2003, el 10\% de los españoles padece esta enfermedad u otros problemas reumáticos ${ }^{4}$, que aumenta en las mujeres hasta el $22 \%$.

Los sintomas más característicos de la artrosis son el dolor y la incapacidad fisica ${ }^{5-8}$, cuya combinación reduce la calidad de vida de los afectados ${ }^{8}$.

El dolor en la articulación afectada es el síntoma más común, y contribuye a descensos significativos en la capacidad funcional de la persona ${ }^{9.10}$. La causa anatómica no está clara y es probable que varíe entre individuos; estudios recientes confirman la heterogeneidad del dolor aıtrósico según localización, severidad, etc ${ }^{10}$.

Incidir sobre los factores de riesgo podría reducir los síntomas y la discapacidad asociados a la artrosis. Muchos autores contemplan el sobrepeso y la obesidad como factores de riesgo, y algunos estudios apuntan a que la reducciôn de los sîntomas se correlaciona en mayor medida con la reducción de la masa grasa que con la reducción del peso corporal total ${ }^{2,8,11}$. También está la 
debilidad del cuádriceps, que es la variable que por sí sola predice en mayor medida la limitación funcional de la extremidad inferior, mâs aun que el dolor ${ }^{3,12}$, que puede ser un factor de riesgo para la artrosis de rodilla $a^{5.8}$. Por otro lado, algunos estudios demuestran que un reducido sentido de la posición contribuye al desarrollo de la artrosis, ademâs de que la propiocepción estâ significativamente disminuida en adultos mayores con artrosis ${ }^{8,12}$.

Por último, y dado el impacto que tiene en el diseño de posibles estudios clinicos, se debe señalar que el factor de riesgo más determinante, tanto para la artrosis radiológica como para la sintomâtica en cualquier articulación, es la edad ${ }^{8}$. A pesar de esto, los sujetos de la mayoría de los estudios analizados más adelante tienen una media de edad en torno a los 65 años, lo cual sólo permite sacar conclusiones para esta edad.

Los tratamientos utilizados en la actualidad no se centran en mejorar el estado del cartílago (hoy por hoy no hay medicamentos que to consigan), sino en tratar el dolor producido por la enfermedad ${ }^{2,12,13}$, además de intentar maximizar la independencia funcional y mejorar la calidad de vida ${ }^{\mathbf{3}}$.

Atendiendo a esta orientación de las terapias y a los factores de riesgo, vemos que un posible tratamiento no farmacológico que puede incidir sobre ellos (y potencialmente reducir los sintomas de la artrosis) es el ejercicio fisico. Este concepto se refiere a todo movimiento corporal realizado con una metodología concreta y persiguiendo un fin definido.

Esta idea de utilización del ejercicio es apoyada por el consenso existente entre organizaciones $\mathrm{e}$ investigadores especializados (AGS, EULAR, OARSI y ACR) al incluir en sus guilas de recomendaciones la utilización del ejercicio como terapia ${ }^{8.14-20}$. Además, los expertos están de acuerdo en que el tratamiento del dolor artrósico debería ser integral y multidisciplinario, que incluya tanto terapia no farmacológica como farmacológica. También afirman que el tratamiento inicial debería ser no farmacológico ${ }^{8}$, lo que hace del ejercicio fisico una importante herramienta en las etapas iniciales de la enfermedad.

De todas formas, las causas por las que se produce dolor en la artrosis no están del todo claras ${ }^{2,21}$, y seguramente hasta que éstas no se definan será dificil precisar el mecanismo por el cual el ejercicio físico reduce estos síntomas.

Por otro lado, la mayoría de los estudios que toman como intervención el ejercicio fisico se han realizado sobre artrosis de rodilla, pero los mismos principios probablemente puedan aplicarse en otras localizaciones ${ }^{2}$.

A pesar de estas recomendaciones y de los numerosos estudios que han obtenido mejoras en el dolor y la capacidad funcional mediante programas de ejercicio físico como tratamiento, existe una falta de información práctica. Es decir, no está tan claro qué tipo de ejercicio utilizar ni las características básicas de los programas (duración, volumen, intensidad, etc.).

\section{Búsqueda bibliográfica sistemática}

Con el fin de obtener todos los estudios de los ûltìmos años en los que se investigó la eficacia del ejercicio físico como tratamiento para la artrosis de rodilla, se realizó una búsqueda bibliográfica en las principales bases de datos del área: PubMed. Dialnet y Sportdiscus. Las frases de búsqueda estuvieron compuestas por los têrminos «ejercicio $O R$ exercise» 0 «actividad fisica $O R$ physical activity» $\mathrm{y}$ 《artrosis rodilla $O R$ knee osteoarthritis». Esta revisión se realizó siguiendo el procedimiento de búsqueda sistemática propuesto por Benito et $\mathrm{al}^{22}$, en el que se crea una frase de búsqueda con palabras clave que se introduce en las principales bases de datos, nacionales e internacionales, del área de estudio. Tras obtener los resultados de las diferentes búsquedas, se realiza la selección de artículos con unos criterios de

inclusión preestablecidos. Las referencias de los artículos seleccionados también se revisan con el fin de obtener nuevos trabajos potencialmente interesantes.

Los criterios de inclusión para seleccionar los estudios clínicos por revisar fueron los siguientes: publicados en los últimos 10 años, es decir, el período comprendido entre 1999 y 2008 , en castellano o inglés, artrosis únicamente en la articulación de la rodilla, con descripción detallada del programa de entrenamiento (debía detallar sus caracteristicas principales: volumen, intensidad, duración y progresión del programa), que tuviese un grupo control que no realizase ejercicio fisico o comparación de los valores antes y después del entrenamiento para un mismo grupo (estudios clínicos), y que contemplara las variables dolor de rodilla y/o la capacidad funcional autopercibida y/o el rendimiento en pruebas de capacidad funcional.

Se rechazaron las publicaciones en las que no fue posible obtener el texto completo así como aquéllas en las que los sujetos recibieron una combinación de tratamientos no farmacológicos o habian recibido tratamiento quìrúrgico.

\section{Estudios seleccionados}

La figura 1 refleja el desarrollo de la búsqueda bibliográfica para este trabajo, donde se detalla el número de artículos encontrados asî como los incluidos y los excluidos.

De todos los artículos encontrados tras la búsqueda bibliográfica, se seleccionó un total de 12 estudios clínicos y una revisión sistemática. Los resultados de todos los estudios clínicos seleccionados, salvo uno ${ }^{1}$, mostraron diferencias significativas respecto a los valores iniciales o respecto al grupo control. Las diferencias se presentaron bien en el dolor de la rodilla, bien en la

Trabajos potencialmente relevantes identificados por búsqueda on-line $(n=1.052)$

Trabajos potencialmente relevantes identificados por búsqueda en referencias $(n=53$ )

Exclusiones:

Fecha de publicación anterior a 10 años $(n=241)$

Sin posibilidad de texto completo $(n=628)$

No estudios clinicos $(n=136$ )

Idiomas diferentes a inglés o castellano $(n=18$ )

Artrosis en localización diferente $(n=12)$

Descripción insuficiente del protocolo $(n=9$ )

No medición de dolor de rodilla o capacidad funcional $(n=7)$

Combinación de tratamientos $(n=8)$

Tratamiento quirúrgico $(n=8)$

Intervención distinta a ejercicio físico $(n=24)$

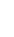

Artículos incluidos en la revisión $(n=13)$

Baker, K.R. y col. (2001)

Deyle, G.D. y col. (2005)

Durmus, D. y col. (2007)

Eyigor, S. y col. (2004)

Gur, $\mathrm{H}$ y col. (2002)

Jan, M.H. y col. (2008)

Lin, D.H. y col. (2007)

\author{
Mangione, K.K. y col. (1999) \\ Messier, S.P. y col. (2000) \\ Roddy, E. y col. (2005) \\ Silva, L.E. y col. (2008) \\ Talbot, L.A. y col. (2003) \\ Topp, R. y col. (2002)
}

Figura 1. Procedimiento de búsqueda sistemático utilizado para la selección de originales. 
capacidad funcional percibida y/o demostrada (mediante pruebas fisicas específicas) o bien en ambos procedimientos a la vez. Además, los resultados de los estudios que compararon dos programas de entrenamiento diferentes no mostraron en ningún caso diferencias entre grupos para el dolor percibido. Sin embargo, en el estudio de Topp et $a^{9}{ }^{9}$ sî se observó una diferencia significativa entre giupos para la subescala del índice WOMAC (Western Ontario and McMaster Universities Osteoarthritis Index), que valora la limitación funcional.

\section{Análisis de los estudios seleccionados}

\section{¿Entrenaniento de fuerza o aeróbico?}

En cuanto a la cualidad principal por desarrollar, encontramos que tanto el ejercicio aeróbico como el ejercicio de fuerza han mostrado su validez en diversos estudios clínicos. Pero, según nuestro conocimiento, únicamente hay una revisión sistemática cuyo objetivo fue comparar la eficacia de los dos tipos de ejercicio de forma indirecta. Según sus resultados, se concluyó que, aunque ambos entrenamientos reducen el dolor y la incapacidad funcional, no hay diferencias en su grado de eficacia ${ }^{23}$. Es decir, ambos programas son igual de efectivos en el tratamiento del dolor sintomático, a falta de una mayor profundización en esta comparación. De esta conclusión derivan las recomendaciones de los organismos de investigación en artrosis, que aconsejan realizar ambos tipos de ejercicios ${ }^{18}$; sin embargo, el entrenamiento con cargas ha demostrado una mayor eficiencia en la ganancia de fuerza y de masa muscular en varones de mediana edad que otras formas de ejercicio 24 .

Ya se ha expuesto que la debilidad muscular es un factor de riesgo y la causa más importante de disfuncionalidad; por lo tanto, el ejercicio de fuerza debería ser imprescindible para los pacientes con artrosis. Poca fuerza en la extremidad inferior se relaciona con un peor equilibrio ${ }^{25}$, y se ha visto que su desarrollo produce una mejora en la propiocepciôn ${ }^{26}$. Por otro lado, el ejercicio aeróbico mejora la capacidad del individuo en tareas que implican la transferencia del peso corporal, como caminar o subir y bajar escaleras $^{12}$.

\section{Caracteristicas generales de los programas}

En la tabla 1 se resumen estas características, en las que hemos incluido los objetivos perseguidos con el entrenamiento, la metodología empleada y los materiales que en su caso se utilizaron, la duración del programa, la localización corporal sobre la que incidió el programa y si hubo o no calentamiento y vuelta a la calma.

\section{Objetivos del programa y metodología}

Básicamente, podríamos reducir los objetivos perseguidos a dos: desartollo de la fuerza (en cualquiera de sus manifestaciones) y aeróbico. En función de estos objetivos y del entorno en el que se desarrolla el programa de ejercicio fisico, encontramos gran variedad de métodos y materiales.

La definición de los objetivos en los estudios clínicos sigue las recomendaciones, tanto para la población en general como para los sujetos que sufren artrosis ${ }^{8,15-19,27}$, que aconsejan desarrollar estas dos cualidades fisicas. A pesar de que el trabajo de estas dos cualidades en los sujetos con artrosis es imprescindible, es necesario tambiên el trabajo de la flexibilidad o la amplitud de movimiento y del equilibrio, cualidades fisicas importantes para mejorar la calidad de vida, evitar caîdas y mejorar la propiocepción de los sujetos ${ }^{28-30}$.

El trabajo de flexibilidad como objetivo por desartollar sólo aparece en dos de los estudios revisados ${ }^{31,32}$; sin embargo, no puede diferenciarse su efecto debido a que el tratamiento se da en conjunto con otras cualidades físicas.

Por otro lado, el equilibrio se trabaja en numerosas ocasiones de forma indirecta al realizar otros ejercicios, pero en los artículos revisados no se encontró un ejercicio que de forma directa persiguiese el desarrollo de esta cualidad, asî como tampoco se menciona su desarrollo en las guías de recomendaciones consultadas.

En cuanto a los métodos utilizados, para el desarrollo de la capacidad aeróbica se han empleado tanto bicicleta estâtica como caminar, aunque este último método se empleó en combinación con ejercicios de fuerza.

Para el desarrollo de la fuerza encontramos que se utilizan ejercicios isométricos ${ }^{9,31-33}$, ejercicios isotónicos en cadena abjerta o cerrada $a^{3.9 .13 .31,32,34-36}$, ejercicios isocinéticos ${ }^{5.13} \mathrm{y}$ electroestimulación ${ }^{1,33}$.

Enfrentando los ejercicios isotónicos e isométricos (los dos métodos más asequibles para la población en general), vemos que la limitación de los estudios que utilizan el entrenamiento isométrico es que la ganancia de fuerza tiene lugar en ángulos articulares pequeños, pero una posible ventaja de este tipo de entrenamiento es que puede no enfrentar a la articulación a tanto estrés ${ }^{\vartheta}$. Por otro lado, se ha visto que el entrenamiento de fuerza dinámico se correlaciona con una mejora en el rendimiento neuromuscular ${ }^{9}$, lo que podría influir positivamente en otro de los factores de riesgo que tambiên sufren los afectados por artrosis: la propiocepción reducida.

Los ejercicios isocinéticos requieren de máquinas especiales, que no son frecuentes en las salas de recuperación, por lo que resultan caros $e$ inaccesibles, $y$ no se han visto mejoras significativamente diferentes de las que producen los ejercicios de fuerza convencionales ${ }^{13}$, to mismo que ocurre con la electroestimulacióo $^{33}$, a pesar de ser más accesible que las máquinas isocinéticas.

Otra cuestión relevante por abordar es la forma de encadenar los ejercicios en el entrenamiento de fuerza, es decir, el método empleado. En este punto encontramos que todos los estudios que detallan este aspecto realizan un programa en forma de series. Una posible linea de investigaciôn, por lo tanto, es comparar los dos tipos de metodologias (en forma de series o de circuitos), dado que inciden de manera diferente en el desarrollo de la fuerza y la hipertrofia y podrian influir en las consecuencias de la intervención.

En los materiales empleados para el desarrollo de los programas de entrenamiento de fuerza tambiên encontramos gran diversidad, aunque los más utilizados son las tobilleras lastradas, las mancuernas y/o las autocargas. Seis de los doce estudios revisados emplean este tipo de material $]^{3,9,13,31,32,36}$.

La utilización de pesos ligeros con tobilleras (de baja intensidad) puede verse justificada por el hecho de que los pacientes con artrosis severas puedan no tolerar el entrenamiento con mayores pesos, pero en dos artículos en los que se emplea un porcentaje de intensidad mayor que el $50 \%$ de la carga para 1 repetición máxima (RM) o para $10 \mathrm{RM}$, se obtuvieron mejoras significativas tanto en dolor como en capacidad funcional ${ }^{13.34}$.

\section{Duración del programa}

La duración de los programas varía desde las 4 semanas empleadas por Deyle et al $^{31}$ hasta los 6 meses utilizados por Messier et $a^{36}$; el valor medio se encuentra en una duración de 
Tabla 1

Características generales de los programas de entrenamiento empleados en los estudios clínicos revisados

\begin{tabular}{|c|c|c|c|c|c|c|c|}
\hline Estudios & Muestra y edad & Objetivos & Duración & Método y fo material & localización & Calentamiento & Vuelta a la calma \\
\hline Silva (2008) & $\begin{array}{l}32 ; 59 \pm 7,6 \text { años } \\
32 ; 59 \pm 6,1 \text { años }\end{array}$ & $\begin{array}{l}\text { Flexibilidad, fuerza } \\
\text { isométrica y } \\
\text { dinámica, mejora de } \\
\text { la marcha }\end{array}$ & 18 semanas & $\begin{array}{l}\text { En agua } \\
\text { En sala }\end{array}$ & Tren inferior & No & No \\
\hline $\operatorname{Jan}(2008)$ & $\begin{array}{l}34 ; 63 \pm 6,6 \text { anัos } \\
32 ; 62 \pm 7,1 \text { años }\end{array}$ & $\begin{array}{l}\text { Fuerza dinámica en } \\
\text { cadena cerrada }\end{array}$ & 8 semanas & $\begin{array}{l}\text { En Dynamic Track Leg } \\
\text { Press Machine, alta } \\
\text { intensidad } \\
\text { En Dynamic Track Leg } \\
\text { Press Machine, baja } \\
\text { intensidad }\end{array}$ & Tren inferior & $\begin{array}{l}10 \text { min de } \\
\text { bicicleta estática }\end{array}$ & $\begin{array}{l}10 \text { min de } \\
\text { aplicación de } \\
\text { frío }\end{array}$ \\
\hline $\operatorname{Lin}(2007)$ & $26 ; 61 \pm 7.7$ aกัos & $\begin{array}{l}\text { Fuerza dinámica en } \\
\text { cadena celrada }\end{array}$ & 8 semanas & $\begin{array}{l}\text { Shuttle Mini Clinic } \\
\text { Resistance Device }\end{array}$ & Tren inferior & No & No \\
\hline Durmus (2007) & $\begin{array}{l}25 ; 55 \pm 2 \text { años } \\
32 ; 55 \pm 1,7 \text { años }\end{array}$ & Fuerza & 4 semanas & $\begin{array}{l}\text { Electroestimulación } \\
\text { Jsometría con } \\
\text { biofeedback }\end{array}$ & Cuádriceps & No & No \\
\hline Deyle (2005) & $68 ; 62 \pm 9$ años & $\begin{array}{l}\text { Fuerza isométrica y } \\
\text { dinámica, } \\
\text { flexibilidad }\end{array}$ & 4 semanas & $\begin{array}{l}\text { Ejecución en casa del } \\
\text { sujeto. Ejercicios de } \\
\text { fuerza convencionales }\end{array}$ & Tren inferior & No & No \\
\hline Talbot (2003) & $18 ; 70 \pm 5.6$ aกัos & Fuerza & 12 semanas & Electroestimulación & Cuádriceps & No & No \\
\hline Gür (2002) & $\begin{array}{l}9: 55 \pm 12 \text { años } \\
8: 56 \pm 12 \text { años }\end{array}$ & Fuerza dinámica & 8 semanas & $\begin{array}{l}\text { Jsocinético concéntrico } \\
\text { Jsocinético } \\
\text { concéntrico-excéntrico }\end{array}$ & $\begin{array}{l}\text { Cuádriceps. } \\
\text { isquiotibial }\end{array}$ & No & No \\
\hline Topp $(2002)$ & $32 ; 55 \pm 1.7$ aйos & $\begin{array}{l}\text { Fuerza isométrica o } \\
\text { dinámica }\end{array}$ & 16 semanas & Ejercicios isométricos & $\begin{array}{l}\text { Musculatura del } \\
\text { tobillo y la } \\
\text { rodilla }\end{array}$ & No & $\begin{array}{l}5 \text { min de } \\
\text { estiramiento }\end{array}$ \\
\hline & $32 ; 55 \pm 1,7$ años & & & $\begin{array}{l}\text { Ejercicios de fuerza } \\
\text { convencionales }\end{array}$ & & $\begin{array}{l}5 \text { min de } \\
\text { movimientos de } \\
\text { piernas }\end{array}$ & \\
\hline Eyigor (2004) & $21 ; 53 \pm 6.7$ años & Fuerza dinámica & 6 semanas & Ejercicio isocinético & Cuádriceps & $\begin{array}{l}10 \text { min de } \\
\text { caminar }\end{array}$ & No \\
\hline & $18 ; 52 \pm 8,1$ aก̆०s & & & $\begin{array}{l}\text { Ejercicios de resistencia } \\
\text { progresiva }\end{array}$ & & & \\
\hline Baker (2001) & $23 ; 69 \pm 6$ años & Fuerza dinámica & 4 meses & $\begin{array}{l}\text { Ejecución en cada } \\
\text { extremidad del sujeto. } \\
\text { Ejercicios de fuerza } \\
\text { convencionales }\end{array}$ & Tren inferior & No & No \\
\hline Messier (2000) & $11 ; 69 \pm 5$ años & $\begin{array}{l}\text { Resistencia } \\
\text { aeróbica, fuerza } \\
\text { dinámica }\end{array}$ & 6 meses & $\begin{array}{l}\text { Ejercicios de fuerza } \\
\text { convencionales }\end{array}$ & $\begin{array}{l}\text { Tren superior e } \\
\text { inferior }\end{array}$ & $\begin{array}{l}5 \mathrm{~min}(\mathrm{no} \\
\text { detalla) }\end{array}$ & $\begin{array}{l}5 \text { min (no } \\
\text { detalla) }\end{array}$ \\
\hline \multirow[t]{2}{*}{$\begin{array}{l}\text { Mangione } \\
(1999)\end{array}$} & 20:71 $\pm 6,2$ años & Resistencia aesóbica & 10 semanas & $\begin{array}{l}\text { Cicloergómetro de baja } \\
\text { intensidad }\end{array}$ & Tren inferior & $\begin{array}{l}\text { Caminar rápido, } \\
\text { estiramientos }\end{array}$ & $\begin{array}{l}\text { Caminar, } \\
\text { ejercicios de } \\
\text { respiración }\end{array}$ \\
\hline & $19: 71 \pm 7.7$ aйо & & & $\begin{array}{l}\text { Cicloergómetro de alta } \\
\text { intesidad }\end{array}$ & & & \\
\hline
\end{tabular}

aproximadamente 11 semanas. A pesar de que el programa de Deyle et al podría parecer estîmulo insuficiente debido a su brevedad, estos autores observaron una mejora significativa, comparando inicio y final del programa, en la distancia caminada en $6 \min$ (el $10 \%$ de mejora) y en el índice WOMAC (el $26 \%$ de mejora $)^{31}$. De todas formas, aun habiêndose observado mejoras con esta intervención tan breve, lo ideal es realizar un programa de duración indefinida, dados los resultados del seguimiento que realizan Talbot et $\mathbf{l}^{1}$ tras la intervención, en los que se observa una pérdida de las mejoras obtenidas con el programa. En relación con la duración, se ha mencionado anteriormente que algunos estudios muestran que los efectos del ejercicio en la altrosis son transitorios y que los resultados empeoran cuando cesa el ejercicioio ${ }^{19}$.

\section{Localización corporal}

Aunque los grupos musculares trabajados difieren entre estudios, la mayoría son muy analíticos. Un tercio de los programas se centra únicamente en el cuádriceps, o en éste y la musculatura isquiotibial ${ }^{1,5,13,33}$, y únicamente un estudio incide tanto en el tren inferior como en el superior ${ }^{36}$.
Si el objetivo del programa de entrenamiento es la mejora localizada de una región anatómica, es posible que sea mucho mâs práctico utilizar un programa analítico, pero no hay que olvidar que hay una fuerte relación de la artrosis con la edad y parece evidente que un programa de trabajo con cargas más general puede aportar ventajas añadidas en la salud ${ }^{28,29}$.

\section{Calentamiento y vuelta a la calma}

No hemos encontrado evidencias sobre la influencia del calentamiento y la vuelta a la calma y los síntomas de la artrosis. Sin embargo, en la población general, en cuanto a la parte inicial, si bien el estiramiento no parece influir en reducir el indice de lesiones entre deportistas ${ }^{37.38}$, el calentamiento sí está relacionado con este hecho ${ }^{39}$.

Encontramos que sólo en 5 de los altículos revisados se menciona que hacen calentamiento ${ }^{9,13,34,36,40}$ y sólo en 4 , la inclusión de una vuelta a la calma $a^{9,34,36,40}$.

En general, los estudios dedican entre 5 y $10 \mathrm{~min}$ para el calentamiento, con igual intervalo para el enfriamiento. Los mêtodos utilizados para calentar son, en su mayoría, aeróbicos, además de incluir en ocasiones estiramientos y movimientos 
articulares ${ }^{9.13 .34 .36 .40}$. Para el enfriamiento los mêtodos difieren

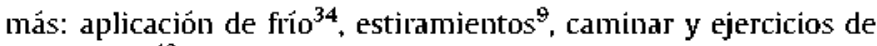
respiración ${ }^{40}$.

\section{Características de la carga de entrenamiento}

Estas características, que se resumen en las tablas 2 y 3 , incluyen información sobre el tiempo de duración de las sesiones, su frecuencia semanal, el volumen de ejercicio realizado y su progresiôn durante el programa (expresado en número de ejercicios, número de series y número de repeticiones), la intensidad del ejercicio y su progresión, los descansos entre ejercicios, series o repeticiones, y el ritmo de ejecución en el caso de ejercicios de fuerza dinámicos.

\section{Duración de la sesión}

Aunque no todos los estudios reflejan este dato, la variabilidad en esta característica es grande, ya que van desde los 15 hasta los 60 min. Son las intervenciones que utilizan la electroestimulación las que tienen las sesiones de menor duración (15-20 min $)^{1,33}$, mientras que en el resto de los programas únicamente utilizan sesiones de menos de 50 min en las fases iniciales.

\section{Frecuencia semanal}

La frecuencia de entrenamiento, salvo en 3 de los estudios revisados ${ }^{13,31,33}$, es siempre de 3 días por semana. Esto no es casualidad, ya que la mayoría de los estudios utilizan las pautas de trabajo del ACSM (American College of Sports Medicine) para las personas mayores, que señala 2 días a la semana como la frecuencia mínima para el entrenamiento de fuerza ${ }^{28,29,41}$. Para el entrenamiento aeróbico, la frecuencia recomendada por este organismo es de 5 dias. Con esta indicación queremos señalar que, aunque los estudios en artrosis han demostrado eficacia con tan sólo 3 días semanales, un mejor diseño del programa debería ir encaminado a mejorar integralmente la salud, por lo que se debería respetar esta frecuencia mínima estipulada.

\section{Volumen inicial y su progresión}

En los entrenamientos de fuerza, el análisis del número de ejercicios por grupo muscular indica que en la mayoría de los programas se emplea un único ejercicio. $Y$ si antes hemos comprobado que la mayoría de los estudios sólo trabajan la articulación implicada, esto podría ser un impedimento para la continuidad en el programa por parte del sujeto (aunque no se refleje en los estudios), ya que un solo ejercicio con una sola articulación trabajada implica una monotonía que podría evitarse

Tabla 2

Características de la carga de entrenamiento de los programas । (estudios de 2003 a 2008 )

\begin{tabular}{|c|c|c|c|c|c|c|c|}
\hline Estudio & Grupo & $\begin{array}{l}\text { Tiempo de } \\
\text { sesión }\end{array}$ & $\begin{array}{l}\text { Frecuencia } \\
\text { semanal }\end{array}$ & $\begin{array}{l}\text { Volumen inicial y } \\
\text { progresión } \\
\text { (ejercicios/series] } \\
\text { repeticiones) }\end{array}$ & $\begin{array}{l}\text { Intensidad inicial y } \\
\text { progresión }\end{array}$ & Descanso & $\begin{array}{l}\text { Ritmo de } \\
\text { ejecución }\end{array}$ \\
\hline Silva et al (2008) & Agua. 5ala & 50 min & 3 dias & $\begin{array}{l}\text { Estiramientos: } 1 \\
\text { serie/2 repeticiones } \\
\text { de } 20 \text { s. Fuerza } \\
\text { isométrica: } 2 / 1 / 7-10 \\
\text { de } 6 \text { s. Fuerza } \\
\text { isotónica ( } 10 \\
\text { ejercicios): semana } \\
\text { 1-3: } 20 \text { repeticiones; } \\
\text { semana } 4: 40 \\
\text { repeticiones }\end{array}$ & $\begin{array}{l}\text { Fuerza isotónica: } \\
\text { semana 1: sin } \\
\text { resistencia; semanas 2- } \\
4: \text { banda elástica o } 1 \mathrm{~kg} \\
\text { de lastre }\end{array}$ & No detalla & No detalla \\
\hline \multirow[t]{2}{*}{ Jan et al (2008) } & $\begin{array}{l}\text { Fuerza de alta } \\
\text { intensidad }\end{array}$ & $30 \mathrm{~min}$ & 3 dias & $1 / 3 / 8$ & $\begin{array}{l}60 \%, 1 \text { RM estimada. } \\
\text { Cada } 2 \text { semanas: retest } \\
\text { de } 1 \text { RM. Aumento del } \\
5 \%\end{array}$ & $\begin{array}{l}1 \text { min entre } \\
\text { series; } 5 \text { min } \\
\text { entre piernas }\end{array}$ & $1-1$ \\
\hline & $\begin{array}{l}\text { Fuerza de baja } \\
\text { intensidad }\end{array}$ & $50 \mathrm{~min}$ & & $1 / 10 / 15$ & $10 \%, 1 \mathrm{RM}$ estimada & & \\
\hline lin et al (2007) & $\begin{array}{l}\text { Fuerza de cadena } \\
\text { cerrada }\end{array}$ & No detalla & 3 dias & $1 / 10 / 10$ & $\begin{array}{l}\text { Inicial: } \mathbf{1 0} \% \text { del peso } \\
\text { corporal. Aumento del } \\
5 \% \text { cada } 2 \text { semanas }\end{array}$ & $\begin{array}{l}1 \mathrm{~min} \text { entre } \\
\text { series; } 5 \text { min } \\
\text { entre piemas }\end{array}$ & $1-1$ \\
\hline \multirow[t]{2}{*}{$\begin{array}{l}\text { Durmus et al } \\
\qquad(2007)\end{array}$} & Electroestimulación & $20 \mathrm{~min}$ & 5 días & $10 \mathrm{~s}$ de contracción & $\begin{array}{l}\text { En aparente contracción } \\
\text { muscular (entre } 70 \mathrm{y} \\
120 \mathrm{~mA} \text { ) }\end{array}$ & 105 & - \\
\hline & $\begin{array}{l}\text { Isometría con } \\
\text { biafeedback }\end{array}$ & & & 1 ejercicio & Máxima contracción & $50 \mathrm{~s}$ & \\
\hline Deyle at el (2005) & $\begin{array}{l}\text { Ejecución en casa del } \\
\text { sujeto }\end{array}$ & No detalla & $\begin{array}{l}7 \text { dias: } \\
\text { flexibilidad; } 3 \\
\text { dias: fuerza }\end{array}$ & $\begin{array}{l}\text { En fuerza ( } 3 \\
\text { ejercicios): } 10 \\
\text { repeticiones o hasta } \\
\text { completal } 30 \mathrm{~s} \text {. } \\
\text { Mantenel } \\
\text { isométricamente } 3 \mathrm{~s} \\
\text { en cada repetición }\end{array}$ & $\begin{array}{l}\text { Autoadministrada. } \\
\text { Aumento de la } \\
\text { intensidad } \\
\text { autoadministrada }\end{array}$ & No detalla & No detalla \\
\hline Talbot et al (2003) & Electroestimulación & 15 min & 3 dias & 10 s de contracción & $\begin{array}{l}\text { Semanas 1-4: } 10-20 \% \text { de } \\
\text { la MCV; semanas } 5-8 \text {; } \\
20-30 \% \text { de la MCV; } \\
\text { semanas } 9-12: 30-40 \% \\
\text { de la MCV }\end{array}$ & $50 s$ & - \\
\hline
\end{tabular}


Tabla 3

Características de la carga de entrenamiento de los programas u (estudios de 1998 a 2002)

\begin{tabular}{|c|c|c|c|c|c|c|c|}
\hline Estudio & Grupo & $\begin{array}{l}\text { Tiempo de } \\
\text { sesiốn }\end{array}$ & $\begin{array}{l}\text { Frecuencia } \\
\text { semanal }\end{array}$ & $\begin{array}{l}\text { Volumen inicial y } \\
\text { progresión (series y } \\
\text { repeticiones) }\end{array}$ & $\begin{array}{l}\text { lntensidad inicial y } \\
\text { progresión }\end{array}$ & Descanso & $\begin{array}{l}\text { Ritmo de } \\
\text { ejecución }\end{array}$ \\
\hline Gür et al (2002) & $\begin{array}{l}\text { Isocinético } \\
\text { concéntrico } \\
\text { Isocinético } \\
\text { concéntrico- } \\
\text { excéntrico }\end{array}$ & No detalla & 3 dias & $\begin{array}{l}12 \text { flexoextensiones de } \\
\text { rodilla, todo concéntrico } \\
6 \text { extensiones } \\
\text { concéntricas y } 6 \\
\text { flexiones excéntricas } \\
\text { (alternativas): } 6 \\
\text { extensiones excéntricas } \\
\text { y } 6 \text { flexiones } \\
\text { concéntricas } \\
\text { (alternativas) }\end{array}$ & $\begin{array}{l}\text { Esfuerzo máximo en un } \\
\text { rango de velocidades } \\
\text { angulares entre } 30^{\circ} / \mathrm{s} \text { y } \\
180^{\circ} / 5\end{array}$ & $\begin{array}{l}5 \text { min entre } \\
\text { piernas } \\
2 \text { min entre } \\
\text { series; } 5 \text { min } \\
\text { entre piernas }\end{array}$ & $\begin{array}{l}\text { Velocidad } \\
\text { angular }\end{array}$ \\
\hline \multirow[t]{2}{*}{ Topp et al (2002) } & $\begin{array}{l}\text { Ejercicios } \\
\text { isométricos }\end{array}$ & 40 min- $1 \mathrm{~h}$ & 3 días & $\begin{array}{l}6 \text { ejercicios/1 serie/8 } \\
\text { repeticiones. Aumento } \\
\text { de volumen cada } \\
\text { semana; semanas } 9-16 \text { : } \\
3 \text { series } 12 \text { repeticiones }\end{array}$ & $\begin{array}{l}\text { Semanas 1-2: tensión } \\
\text { media; tras semana } 2 \text { : } \\
\text { tensión máxima }\end{array}$ & $\begin{array}{l}\text { Semanas } 9-16 \text { : } \\
2 \text { min entre } \\
\text { series }\end{array}$ & - \\
\hline & $\begin{array}{l}\text { Ejercicios } \\
\text { dinámicos }\end{array}$ & & & $\begin{array}{l}6 \text { ejercicios } 1 \text { serie/8 } \\
\text { repeticiones de } 3-5 \mathrm{~s}\end{array}$ & $\begin{array}{l}\text { Semanas 1-2: fatiga } \\
\text { media; semanas } 9-16 \text {; } \\
\text { fatiga moderada }\end{array}$ & & No detalla \\
\hline \multirow[t]{2}{*}{ Eyigor et al (2004) } & $\begin{array}{l}\text { Ejercicio } \\
\text { isocinético }\end{array}$ & No detalla & 3 dias & $\begin{array}{l}1 \text { ejercicio/ } 4 \text { velocidades } \\
\text { angulares } / 3 \text { series } / 6 \\
\text { repeticiones }\end{array}$ & $\begin{array}{l}\text { Esfuerzo máximo a } 60^{\prime} / \mathrm{s} \text {, } \\
90^{\prime \prime} / \mathrm{s}, 120^{\prime} / \mathrm{s} \text { y } 180^{\prime} / \mathrm{s}\end{array}$ & $\begin{array}{l}20 \text { s entre } \\
\text { series }\end{array}$ & $\begin{array}{l}\text { Velocidad } \\
\text { angular }\end{array}$ \\
\hline & $\begin{array}{l}\text { Ejercicio de } \\
\text { resistencia } \\
\text { progresiva }\end{array}$ & & 5 días & $\begin{array}{l}1 \text { ejercicio/3 serjes/10 } \\
\text { repeticiones con } 55 \\
\text { isométricos en cada } \\
\text { repetición }\end{array}$ & $\begin{array}{l}1{ }^{\mathrm{a}} \text { serie: } 50 \% \text { de } 10 \mathrm{RM} \\
2 .^{\mathrm{a}} \text { serie: } 75 \% \text { de } 10 \mathrm{RM} \\
3 .^{\text {a }} \text { serie: } 100 \% \text { de } 10 \mathrm{RM} \text {. } \\
\text { Restest de } 10 \mathrm{RM} \\
\text { semanales }\end{array}$ & No detalla & $\begin{array}{l}\text { Movimien-to } \\
\text { lento }\end{array}$ \\
\hline Baker et al (2001) & $\begin{array}{l}\text { En casa del } \\
\text { sujeto }\end{array}$ & No detalla & 3 dias & $\begin{array}{l}7 \text { ejercicios } / 2 \text { series } / 12 \\
\text { repeticiones }\end{array}$ & $\begin{array}{l}3-5 \text { en escala de Borg de } \\
10.1 .: \text { aumentar a } 8 \text { en } \\
\text { Borg; } 20^{\circ} ; \text { aumento de } 1 \\
\text { lb en cada pierna cuando } \\
\text { el esfuerzo es menor de } 6 \\
\text { o se realizan más de } 12 \\
\text { repeticiones }\end{array}$ & No detalla & No detalla \\
\hline Messier et al (2000) & En sala & $1 \mathrm{~h}$ & 3 dias & $\begin{array}{l}\text { Aeróbico: } 2 \text { series de } \\
\text { 10min caminando. } \\
\text { Fuerza: } 7 \text { ejercicios } 11 \\
\text { serie/ } 10-12 \text { repeticiones }\end{array}$ & $\begin{array}{l}\text { Aeróbico: } 50-75 \% \text { de la } \\
\text { reserva de la frecuencia } \\
\text { cardíaca. Fuerza: no } \\
\text { detalla }\end{array}$ & $\begin{array}{l}\text { Fuerza: } \\
\text { 1-1.5 min entre } \\
\text { ejercicios }\end{array}$ & No detalla \\
\hline $\begin{array}{l}\text { Mangione et al } \\
\text { (1999) }\end{array}$ & $\begin{array}{l}\text { Cicloergómetro } \\
\text { de baja } \\
\text { intensidad } \\
\text { Cicloergómetro } \\
\text { de alta } \\
\text { intensidad }\end{array}$ & $1 \mathrm{~h}$ & 3 dias & $\begin{array}{l}25 \text { min de pedaleo } \\
\text { continuo }\end{array}$ & $\begin{array}{l}40 \% \text { de la reserva de la } \\
\text { frecuencia cardiaca, sin } \\
\text { resistencia en las ruedas } \\
70 \% \text { de la reserva de la } \\
\text { frecuencia cardiaca, sin } \\
\text { resistencia en las ruedas }\end{array}$ & - & - \\
\hline
\end{tabular}

RM: repetición máxima.

al aumentar y variar el número de ejercicios a medida que avanza la experiencia del sujeto y su estado de forma.

La cantidad de series utilizadas es entre 1 y 10 , sin mostrar un patrón común entre estudios. En cuanto al número de repeticiones, lo más frecuente es encontrar estudios con 8 a 12. Aquí volvemos a encontrar coincidencia con las recomendaciones del ACSM, lo que hace de ésta otra pauta por seguir ${ }^{27,29}$.

En cuanto a la progresión, el aumento de series y repeticiones se emplea sólo en dos estudios ${ }^{9.32}$. A pesar de que una progresión en el número de series y repeticiones es necesaria para una correcta adaptación ${ }^{42}$, no se observa esta pauta en los estudios ni está recomendada su utilización en las revisiones sobre el tema $a^{2,8,7,15-19}$.

\section{[ntensidad inicial y su progresión}

En los programas de entrenamiento aeróbico, la intensidad empleada estâ entre el 40 y el $75 \%$ de la reserva de la frecuencia cardiaca $^{36.40}$. Es decir, una intensidad moderada-alta, que permitiô la obtenciôn de diferencias significativas con respecto a los valores iniciales, a pesar de que en ningún caso se programó una progresiôn en intensidad. En ambos casos se obtuvieron también diferencias significativas en cuanto al dolor y la capacidad física, por lo que puede emplearse este grado de intensidad sin perjudicar los sintomas de la artrosis. Es necesario aclarar en este punto que, en el caso de la utilización de cicloergómetros, la intensidad requerida se alcanzó siempre sin emplear resistencia al pedaleo, sino aumentando su cadencia.

Aunque la intensidad muestra una gran heterogeneidad entre los estudios, los autores muestran cargas moderadas entre el $60 \%$ de $1 \mathrm{RM}^{34}$ y del 50 al $100 \%$ de $10 \mathrm{RM}^{13}$.

Sólo tres de los doce estudios revisados no programaron un aumento de intensidad ${ }^{5,13,33}$, dado que desde el inicio del programa requieren una contracción máxima. El resto de los programas progresa de forma muy diferente; desde el empleo de escalas subjetivas o percepciones del esfuerzo hasta la utilizaciôn de materiales para añadir resistencia, como mancuernas o bandas elásticas. En este sentido, no encontramos un patrón común de progresión, y puesto que todos los programas han obtenido resultados favorables, podemos decir que es correcta cualquier progresiôn que respete la adaptación del propio sujeto, a pesar de que la progresión en algunos casos parece excesivamente cautelosa $\mathbf{a}^{9,31}$. 
Tabla 4

Características del programa estándar para el tratamiento de la artrosis de rodilla

Objetivo

Metodología

Materiales

Duración del proğama

Localización corporal

Calentamiento y vuelta a la calma

Duración de la sesión

Frecuencia semanal

Volumen inicial y progresión

lntensidad inicial y progresiól

Descansos

Ritmo de la ejecución
Fuerza dinámica

Ejercicios de autocargas

Tobilleras lastradas y mancuernas

11 semanas, aunque recomendamos realizar un programa con duración indefinida para evitas la pérdida de las mejoras obtenidas con el ejercicio.

Tren inferior, aunque deben trabajarse todos los grupos musculares importantes.

Calentamiento: métodos aeróbicos para el calentamiento. Vuelta a la calma: en función del efecto del entrenamiento. hielo. estiramientos o ejercicios de respiración; $5 \mathrm{~min}$ para cada uno 50 min- $1 \mathrm{~h}$

3 dias

Ejercicios: 1 por grupo muscular. Series y repeticiones: 1 serie, 8-10 repeticiones. Progresión: sin progresión, aunque recomendamos aumentar el número de ejercicios realizados por grupo musculas para evitar la monotonía y el abandono del programa.

Ljgera-moderada, en torno al 60\% de 1 RM o 50-100\% de 10 RM. Progresión: debe respetar la capacidad de adaptación del sujeto.

50 5- 1 min entre series y 5 min entre ejercicios

Medio-lento

RM: repetición máxima.

En este sentido, también debemos señalar el papel que tiene el dolor articular en la progresión de la carga por vencer. Algunos artículos de revisión y guías de recomendaciones abordan este tema y aconsejan que, cuando el dolor aumenta de forma mantenida tras el ejercicio, el peso en la siguiente sesión ha de reducirse ${ }^{9,43,44}$.

\section{Descansos}

Aunque no todos los estudios describen este dato, podemos identificar un intervalo mâs o menos constante para los que sî lo detallan: entre 50 s y 1 min para el descanso entre las series y 5 min entre ejercicios o piernas.

Ritmo de ejecución

Este concepto se refiere a la velocidad con la que se realizan las fases concéntricas y excéntricas de los ejercicios de fuerza dinámicos. En el caso de los ejercicios isocinéticos, el ritmo está determinado por la velocidad angular empleada. Entre los demâs estudios, únicamente tres trabajos detallan este aspecto; dos de ellos emplean un ritmo 1-1 (1 s para realizar la parte concéntrica del ejercicio y $1 \mathrm{~s}$ para realizar la parte excêntrica) ${ }^{34.35}$, y el otro simplemente señala que ha de realizarse el movimiento de forma lenta ${ }^{13}$.

Los efectos que el ritmo de ejecución tiene en la masa muscular han sido descritos previamente ${ }^{45.46}$ : se observa que, en igualdad de condiciones del resto del entrenamiento, una velocidad más elevada produce un mayor incremento en la masa muscular y en la fuerza ${ }^{45}$.

\section{Conclusiones}

Todos los estudios clínicos, salvo uno, mostraron diferencias significativas en el dolor de la rodilla, en la capacidad funcional percibida y/o demostrada (mediante pruebas fisicas específicas) 0 en ambas variables a la vez.

Tras la revisión se concluyen las características de un programa estándar para el tratamiento de la artrosis de rodilla. Éstas se detallan en la tabla 4 y se han seleccionado con el criterio de mayor concordancia entre los estudios revisados.

El programa estândar descrito tiene las limitaciones derivadas de la heterogeneidad de los estudios seleccionados. Con la evidencia científica de estudios más sólidos en su diseño se podria llegar a conclusiones distintas.

A la hora de describir los programas de entrenamiento empleados, gran parte de los autores obvia elementos cruciales para la definición del programa, con lo que resulta imposible aplicar dichos programas con un objetivo terapéutico o de contrastar los resultados en otro tipo de población.

Futuras líneas de investigaciôn en este campo son la comparaciôn de los diferentes métodos y tipos de entrenamiento en una intervención a largo plazo, además de intentar comprobar el tipo de fuerza más beneficiosa para la enfermedad y los contenidos más adecuados de un programa de ejercicio fisico para el tratamiento de la artrosis de rodilla. Por otro lado, se necesitan estudios con mayor intervalo de edad que permitan obtener conclusiones en grupos de edad avanzada.

\section{Bibliografía}

1. Talbot LA, Gaines JM, Ling 5M, Metter EJ. A home-based protocol of electrical muscle stimulation for quadriceps muscle strength in older adults with osteoarthritis of the knee. J Rheumatol. 2003:30:1571-8.

2. Creamer P. Osteoarthritis pain and its treatment. Curr Opin Rheumatol. 2000;12:450-5

3. Baker KR, Nelson ME. Felson DT, layne JE, Samo R, Roubenoff R. The efficacy of home based progressive strength training in older adults with knee osteoarthritis: A randomized controlled trial. J Rheumatol. 2001;28: 1655-65.

4. Ministerio de Sanidad y Consumo. Encuesta Nacional de Salud 2003 lcitado 25 May 2006]. Disponible en: URL: http://www.msc.es/estadEstudios/estadisticas/encuestaNacional/home.htm

5. Gur H, Cakin N, Akova B, Okay E, Kucukoglu S. Concentric versus combined concentric-eccentric isokinetic training: Effects on functional capacity and symptoms in patients with osteoarthrosis of the knee. Arch Phys Med Rehabil. 2002:83:308-16.

6. Talbot LA, Gaines JM, Huymh TN, Metter EJ. A home-based pedometel-driven walking program to increase physical activity in older adults with osteoarthritis of the knee: A preliminary study. I Am Geriatr Soc. 2003:51:387-92.

7. Manek NJ. Lane NE. Osteoarthritis: Current concepts in diagnosis and management. Am Fam Pliysician. 2000;61:1795-804.

8. American Gerjatrics Society. Exercise prescription for older adults with osteoarthritis pain: Consensus practice recommendations. A supplement to the AGS Clinical Practice Guidelines on the management of chronic pain in older adults. J Am Geriatr Soc. 2001:49:808-23.

9. Topp R, Woolley S, Hornyak $3^{\text {rd }}$, Khuder S, Kahaleh B. The effect of dynanic versus isometric resistance training on pain and functioning among adults with osteoarthritis of the knee. Arch Phys Med Rehabil. 2002:83:1187-95.

10. Brandt K. Ostetoarthritis. Curr Opin Rheumatol. 2000;12:449.

11. Toda Y. Toda T. Takemul'a S. Wada T. Morimoto T, Ogawa R. Change in body fat, but not body weight or metabolic correlates of obesity. is related to symptomatic relief of obese patients with knee osteoarthritis after a weight control program. J Rheumatol. 1998;25:2181-6.

12. Baker $\mathrm{K}, \mathrm{McAlindon} \mathrm{T}$. Exercise for knee osteoarthritis, Curr Opin Rheumatol. $2000 ; 12: 456-63$. 
13. Eyigor S. Hepguler 5. Capaci K. A comparison of muscle training methods in patients with knee osteoarthritis. Clin Rheumatol. 2004;23:109-15.

14. Pendleton A, Alden N, Dougados M, Doherty M, Bannwarth B. Bijlsma JW, et al EULAR recommendations for the management of knee osteoarthritis: Report of a task force of the Standing Committee for International clinical studies lncluding Therapeutic Trials (ESCISIT). Alnn Rheum Dis. 2000;59:936-44.

15. Jordan KM, Arden NK, Doherty M, Bannwarth B, Bijlsma JW, Dieppe $\mathrm{P}$, et al EULAR Recommendations 2003: An evidence based approach to the management of knee osteoarthritis: Report of a task force of the Standing Committee for International Clinical Studies Including Therapeutic Trials (ESCISIT). Ann Rheum Dis. 2003;62:1145-55.

16. Zhang W, Moskowitz RW, Nuki $G$, Abramson S, Altman RD, Arden N, et al. OARSI recommendations for the management of hip and knee osteoarthritis. part I: Critical appraisal of existing treatment guidelines and systematic review of current research evidence. Osteoarthritis Cartilage. 2007;15:981-1000.

17. Zliang W. Moskowitz RW. Nukj G, Abramson S, Altman RD. Arden N, et al. OARSI recommendations for the management of hip and knee osteoarthritis, Part II: OARS] evidence-based, expert consensus guidelines. Osteoarthritis Cartilage. 2008; 16:137-62.

18. Roddy E, Zhang W, Doherty M, Arden NK, Barlow J, Birrell F, et al. Evidencebased recommendations for the role of exercise in the management of osteoarthritis of the hip or knee-the MOVE consensus. Rheumatology (Oxford). 2005:44:67-73.

19. Vignon E, Valat JP. Rossignol M Avouac B, Rozenberg 5, Thoumie P, et al Osteoarthritis of the knee and hip and activity: A systematic international review and synthesis (OASIS). Joint Bone Spine. 2006;73:442-55.

20. National Collaborating Centre for Chronic Conditions. Osteoarthritis: National clinical guideline for care and management in adults. London: Royal College of Pliysicians; 2008.

21. Hunter DJ, McDougall JJ, Keefe FJ. The symptoms of osteoarthritis and the genesis of pain. Rheum Dis Clin North Am. 2008;34:623-43.

22. Benito PJ, Díaz V, Calderón FJ, Peinado AB, Martín C, Álvarez M, et al. La revisión bibliográfica sistemática en fisiología del ejercicio: recomendaciones prácticas. RYCIDE. 2007;3:1-11.

23. Roddy E, Zhang W. Doherty M. Aerobic walking or strengthening exercise for osteoarthritis of the knee? A systematic review. Ann Rheum Dis. 2005:64:544-8

24. Izquierdo M. Hakkinen K. Ibanez J. Kraemer WJ. Gorostiaga EM. Effects of combined resistance and cardiovascular training on strength, power, muscle cross-sectional area, and endurance markers in middle-aged men. Eur J Appl Pliysiol. 2005:94:70-5

25. Messier SP, Glasser JL, Ettinger Jr WH, Craven TE, Miller ME. Declines in strength and balance in older adults with chronic knee pain: A 30-month longitudinal, observational study. Arthritis Rheum. 2002;47:141-8.

26. Hurley MV, Scott DL. Improvements in quadriceps sensorimotor function and disability of patients with knee osteoarthritis following a clinically practicable exercise regime. Br J Rheumatol. 1998;37:1181-7.

27. ACSM. American College of Sports Medicine Position Stand. The recommended quantity and quality of exercise for developing and maintaining cardiorespiratory and muscular fitness, and flexibility in healthy adults. Med Sci Sports Exerc. 1998;30:975-91.

28. Nelson ME, Rejeski W], Blair SN, Duncan PW, Judge JO, King AC, et al. Physical activity and public health in older adults: Recommendation from the American College of 5 ports Medicine and the American Heart Association. Circulation. 2007;116:1094-105.
29. Nelson ME. Rejeski WJ. Blair SN, Duncan PW. Judge JO. King AC. et al. Plyysical activity and public health in older adults: Recommendation fom the American College of 5 ports Medicine and the American Heart Association. Med $5 \mathrm{ci}$ Sports Exerc. 2007;39: 1435-45.

30. Rao SS. Prevention of falls in older patients. Am Fam Physician. 2005;72:81-8.

31. Deyle GD, Allison SC, Matekel Rl Ryder MG, Stang JM, Gohdes DD, et al Physical therapy treatment effectiveness for osteoarthritis of the knee: $A$ randomized comparison of supervised clinical exercise and manual therapy procedures versus a home exercise program. Plys Ther. 2005;85:1301-17.

32. Silva LE, Valim V, Pessanha AP, Oliveira LM, Myamoto S, Jones A, et al. Hydrotherapy versus conventional land-based exercise for the management of patients with osteoarthritis of the knee: A randomized clinical trial. Phys Ther 2008:88:12-21.

33. Durmus D. Alayli G. Canturk F. Effects of quadriceps electrical stimulation program on clinical parameters in the patients with knee osteoarthritis. Clin Rheumatol. 2007:26:674-8.

34. Jan $\mathrm{MH}$, Lin Jl, liau Jl, lin VF, Lin $\mathrm{DH}$ Investigation of clinical effects of high and low-resistance training for patients with knee osteoarthritis: A randomized controlled trial. Phys Ther. 2008;88:427-36.

35. Lin DH, lin VF, Chai HM, Han VC, Jan MH. Comparison of proprioceptive functions between computerized proprioception facilitation exercise and closed kinetic chain exercise in patients with knee osteoarthritis. Clin Rheumatol. 2007;26:520-8.

36. Messier 5P, Loeser RF, Mitchell MN, Valle G, Morgan TP, Rejeski WJ, et al. Exercise and weight loss in obese older adults with knee osteoarthritis: A preliminary study. J Am Geriatr 5oc. 2000;48:1062-72.

37. Hart l. Effect of stretching on sport injury risk: A review. Clin J 5port Med. $2005 ; 15: 113$.

38. Thacker 5B. Gilchrist J. 5troup DF, Kimsey Jr CD. The impact of stretching on sports injury risk: A systematic review of the literature. Med Sci Sports Exerc 2004:36:371-8.

39. Fradkin AJ, Gabbe BJ, Cameron PA. Does warming up prevent injury in sport? The evidence from randomised controlled trials?. J Sci Med Sport 2006;9:214-20.

40. Mangione KK, McCully K, Gloviak A, lefebvre I, Hofmann M, Craik R. The effects of high-intensity and low-intensity cycle ergometry in older adults with knee osteoarthritis. J Gerontol A Biol Sci Med Sci. 1999;54:M184-90.

41. Taylor NF, Dodd KJ. Damiano DL. Progressive resistance exercise in pliysical therapy: A summary of systematic reviews. Phys Ther, 2005;85:1208-23.

42. Baechle TR, Earle RW. Essentials of strength training and conditioning. $2{ }^{\mathrm{a}} \mathrm{ed}$. N5C Association, editor. Champaig: Human Kinetics; 2000.

43. Ramos Álvarez J, López-Silvarrey Varela F, Segovia Martínez l, Montoya Miñano J. Legido Arce J. Prescripción del ejercicio en pacientes con artrosis. Recomendaciones actuales. Selección. 2006;15:144-53.

44. Foley A, Halbert l, Hewitt $T$, Crotty $M$. Does hydrotherapy improve strength and physical function in patients with osteoarthritis-a randonised controlled trial comparing a gym based and a hydrotherapy based strengthening programme. Amn Rheum Dis, 2003;62:1162-7.

45. Shepstone TN, Tang JE, Dallaire S, Schuenke MD, Staron RS, Phillips SM. Shortterm high- vs. low-velocity isokinetic lengthening training results in greater hypertrophy of the elbow flexors in young men. J Appl Physiol. 2005:98: 1768-76.

46. Tanimoto $M$, Ishii $N$. Effects of low-intensity resistance exercise with slow movement and tonic force generation on muscular function in young men. Appl Physiol. 2006: 100:1150-7. 